upf. $\begin{array}{ll}\text { Universitat } \\ \text { Pompeu Fabra } \\ \text { Barcelona }\end{array} \quad \begin{aligned} & \text { Department } \\ & \text { of Economics and Business }\end{aligned}$

Economics Working Paper Series

Working Paper No. 1584

\title{
Teaching microeconomic principles with smartphones - lessons from classroom experiments with classEx
}

Marcus Giamattei and Humberto Llavador 


\title{
Teaching Microeconomic Principles with Smartphones - Lessons from classroom experiments with classEx*
}

\author{
Marcus Giamattei ${ }^{\dagger \S}$ and Humberto Llavador ${ }^{* * \dagger \dagger}$
}

October 2017

\begin{abstract}
Classroom experiments as a teaching tool increase understanding and especially motivation. Traditionally, experiments have been run using pen-and-paper or in a computer lab. Pen-and-paper is time and resource consuming. Experiments in the lab require appropriate installations and impede the direct interaction among students. During the last two years, we have created fully elaborated packages to run a complete course in microeconomics principles using face-to-face experiments with mobile devices. The experiments are based on Bergstrom-Miller (2000), and we used classEx, a free online tool, to run them in the classroom. The packages were used at Universitat Pompeu Fabra with over 500 undergraduate students in the fall 2016. This paper presents our experience on classEx and the Bergstrom-Miller approach working in combination, and the lessons learned.
\end{abstract}

Keywords: experiential learning; microeconomics; mobile devices; classroom experiments; classEx

JEL-Classification: A22, C72, C90, D00

\footnotetext{
* We are grateful to Ted Bergstrom, Johann Graf Lambsdorff, John Millner and Rosemarie Nagel for all their support and extremely helpful suggestions. We thank Katharina Werner and Susanna Grundmann for their feedback. We also want to thank the many instructors who have participated over the years in testing and discussing the experiments and the classEx packages at Pompeu Fabra University and at the University of Alicante. We are especially indebted to Carmen Beviá, Nagore Iriberri and Gianmarco León for their time and contributions. Finally, we thank Thomas Woiczyk for his excellent work in the programing of the classEx packages. The project was partly funded by a PlaCLIK grant offered by Pompeu Fabra University.

${ }^{\dagger}$ Assistant Professor at the Chair of Economic Theory. University of Passau, Germany.

* External Fellow at the Center for Decision Research and Experimental Economics (CeDEx), University of Nottingham, UK

$\S$ Corresponding author: marcus.giamattei@uni-passau.de, Innstr. 27, 94032 Passau, Germany. Telephone 0049851 509 2553, Fax 00498515092552.

${ }^{* *}$ Department of Economics, Pompeu Fabra University.

${ }^{\dagger}$ Affiliate Professor of the Barcelona Graduate School of Economics
} 


\section{INTRODUCTION}

Economic ideas come to life in the classroom for students who take part in experimental markets. Instead of simply learning the theory as dogma to be memorized, students who have just participated in a market and seen the market data are interested to discover for themselves how well (or poorly) the theory works to explain the reality that they have observed. They also observe how heterogeneous students act and the adjustment process to equilibrium, aspects difficult to convey in a lecture.

Classroom experiments have been successfully pursued with the use of pen-and-paper instructions and written contracts (e.g. Frank 1997; Emerson and Taylor 2004, 2007, 2010; Dickie 2006; Durham, McKinnon, and Schulman 2007). Running classroom experiments with pen-and-paper is a time and resource consuming approach. Many instructors who are intrigued by the idea of running classroom experiments are deterred by the organizational burden of running such experiments. Running experiments over the internet is also possible, but lacks the excitement of face-to-face interaction among trading students. These drawbacks disappear when experiments can be run in the classroom using mobile devices such as smartphones, laptops, and netbooks, which are widely available nowadays.

To make classroom experiments a meaningful part of the learning experience, it is crucial to link these experiments with constructive homework, making students reflect on the experiment and analyze the data (Cartwright and Stepova 2012). Interaction in lectures should support comprehension, application and analysis (Dangel and Wang 2008). Bergstrom and Miller (2000) provide a collection of pen-and-paper experiments within an introductory course to microeconomic principles. Experiments are designed such to allow and motivate discussions before, during and after the experiment. Each experiment is accompanied by a set of constructive homework that students must work out before any exposure to a theory class. This way, students are invited to discover the main concepts by themselves and avoid becoming passive receivers. The theory class extends the concepts to more general settings and answers unresolved issues.

Experiments increase both teachers and students enjoyment of the class, engaging with the material more comprehensively and asking more advanced questions (Gremmen and Potter 1997; Ball and Eckel 2004; Ball, Eckel and Rojas 2006) and resulting in higher student motivation (Gremmen and van den Brekel 2013). In our experience, most instructors get excited when introduced to the use of experiments as a teaching tool. However, the adoption of experiments in the classroom, although growing, is still far from being dominant. Why is that? Typically, the main reason is the perception that transitioning to a different teaching methodology will be costly in preparation time and/or installations and material. Our project aims at reducing these costs.

For the last two years, we have created fully elaborated packages in classEx, a free online tool for running experiments in the classroom, to teach a complete course in microeconomics principles based on the Bergstrom-Miller (2000) approach. Each package provides a ready-to-use experiment in classEx, its related homework and the main reference text, as well as an instructor manual and all the material for students. After each experiment, classEx provides information on participation, profits and transactions. A spreadsheet automatically calculates the answers to the 
homework from the output data file in classEx. Results from previous experiments are also available in the repository of classEx.

The Introduction to Microeconomics packages in classEx have been used at Universitat Pompeu Fabra with over 500 undergraduate students since the fall 2016, with a pilot in the fall 2015 . $^{7}$ Students were organized in groups of 25 to 45 students for the face-to-face experiments, and then pulled together in larger groups from 80 to 200 students for the lectures. During the experiments, students move around the classroom, bargaining prices or wages and discussing results. Table 1 in the Appendix presents a list of all experiments used in the course at Universitat Pompeu Fabra with a brief description of each.

This paper describes classEx (Section 1); the Bergstrom-Miller approach to teaching microeconomic principles with experiments (Section 2); and the knowledge obtained from the experience at Universitat Pompeu Fabra in the fall 2016 (Section 3).

\section{INTERACTIVE EXPERIMENTS WITH CLASSEX}

classEx (Giamattei and Lambsdorff 2015) is a web-based online tool for running classroom experiments. It is available free of charge to any lecturer and participant. classEx runs as a centralized application where lecturers and students $\log$ in via their browser. This allows an easy access, as it does not require any installation of apps or programs, facilitating the participation in the experiment. It only requires an internet connection and a standard (up-to-date) browser. ${ }^{8}$ Since classEx is platform-independent, it can be equally used with smartphones, tablets, notebooks or computers. Henceforth, we will refer to them as mobile devices. Instructions, any public information and the generated data while running the experiment can be projected in the classroom to all students. Private information and decisions are displayed on the students' mobile devices. Figure 1 displays an overview

The classEx environment includes three principles: play, create, and share. Lecturers can start right away and play any of the pre-programmed games, including public good games, ultimatum games, coordination games, and guessing games, among others. The catalogue of classEx offers a broad range of games for teaching microeconomics, macroeconomics, game theory, psychology, and other subjects. All games for the microeconomics principles course described in this paper are readily available in classEx.

of a standard setting in the classroom.

\footnotetext{
7 The packages have already been adopted by other universities in the UK, Spain and Germany (like University of Alicante, University of Hamburg, Universidad Autonoma de Barcelona, Techinal University Clausthal, University of Kassel, University of Gießen and University of Nottingham)

${ }^{8}$ Experiments may be run completely anonymous. Participants do not need to register or to input any personal data. The instructor sets an access password for all and, if wanted, may keep records with an identifying code or alias.
} 


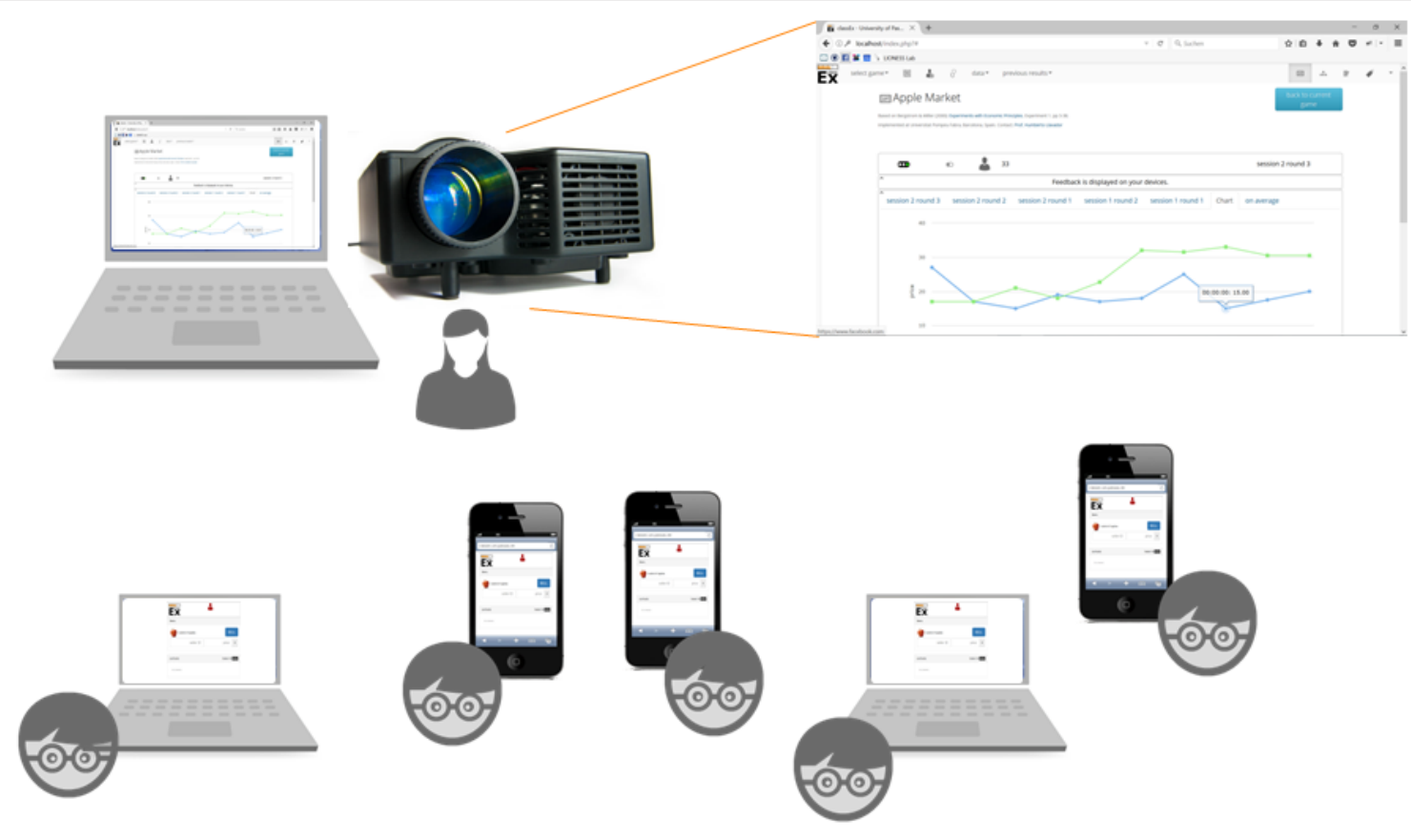

Figure 1. Illustration of the setting in a classroom experiment with classEx. The lecturer projects the overview screen with the game flow and public information. Participants log-in and submit their decisions through their mobile devices.

Besides running pre-programmed games, lecturers can also create their own games and modify existing ones. ${ }^{9}$ This provides instructors with the flexibility to adapt any game to their needs, or use existing games as a starting point for the design of new ones. Instructors can choose among different types of matching, role assignments and between-subject treatments. Games can also be designed with a backend system that only requires the combination of different elements, reducing the need for programming. For more complex setups, on the other hand, code snippets with PHP language allow flexible adjustment.

Thirdly, instructors can share their games and results in classEx. Games can be published in the repository and used by other instructors. Anyone using a game already played by someone else can also access the data obtained in previous sessions. This is an important feature that allows gaining an idea of the expected results prior to running the experiment, facilitating the preparation of the lecture. Additionally, if an instructor obtains unusual outcomes because of misunderstandings or unexpected behaviors, previous results can be used to guarantee meeting the teaching goals set for the lecturer. ${ }^{10}$

\footnotetext{
9 A complete documentation of classEx can be found at www.classEx.uni-passau.de/wiki. To get login credentials go to www.classEx.de

${ }^{10}$ Unusual outcomes should not be automatically discarded. On the contrary, understanding what generated those results usually provides excellent opportunities for an enriching debate. For instance, in small groups, a perfectly
} 
Finally, classEx allows for in-class experiments with face-to-face interaction, even for large groups, while avoiding the problems usually associated with them: time-consuming, record keeping, entering of experimental data... In-class experiments have many benefits, in contrast to online or in-the-lab experiments. Firstly, in-class experiments allow for immediate and personal instructor feedback and a live discussion among students. Secondly, in-class experiments result in face-to-face interaction and physical activity, whose value is supported by the evidence in the literature. ${ }^{11}$ Finally, students report greater satisfaction with in-class experiments and report having more interactions with their classmates and direct contact with a larger number of them (Carter and Emerson 2012). These interactions may foster a greater sense of community and, in our experience, often lead to out of class interactions, like study groups or groups of friends.

\section{THE BERGSTROM-MILLER APPROACH AND THE MATERIAL FOR A COURSE IN MICROECONOMIC PRINCIPLES USING CLASSEX}

This section describes our teaching proposal, which combines elements of a flipped-classroom and experiential learning with the use of experiments in the classroom. Our proposal is based on Bergstrom and Miller's (2000) textbook, and so we coin it the Bergstrom-Miller approach. Each topic is structured in three stages (Figure 2). First, experiments are run in small groups and become the key-learning tool. Each experiment is preceded by a preparation phase with a few warm-up questions that get students thinking about how to behave in the experiment for all possible roles (for example those of sellers and buyers). Students should work on this preparation before coming to the classroom. A discussion during and after the experiment invites students to come up with possible explanations for the observed results and to work on their economic intuitions. Next, students work on a series of constructive homework using the data obtained in the experiment. The homework aims at guiding students to discover the main concepts by themselves before attending the lecture. Finally, the instructor generalizes the analysis (for example by extending it to large economies with a continuum of agents), provides applications, and solves the problems that students had while working on the homework. The approach emphasizes the importance to furnish classroom experiments with constructive homework, making students reflect on the experiment and analyze the data (Cartwright and Stepova 2012).

For a course in microeconomic principles, the basic material is provided in Bergstrom and Miller (2000), a textbook designed to use economic experiments in the classroom. Bergstrom and Miller's (2000) book is comprehensive in providing all the necessary material: a textbook, homework, and an instructor manual containing detailed information on running the experiments. This material has been complemented with classEx packages to run the experiment

competitive market experiment may yield prices that are too high if sellers manage to collude, even though the instructions of the experiment aim at avoiding such behaviour. This "unusual" result can be used to discuss the need for antitrust authorities.

${ }^{11}$ There are many studies where students involved in computer-based or distance learning report missing the face-to-face interaction with their peers and the instructors (Carter and Emerson 2012, 6). 
and to collect the data; with pre-experiment quizzes to test students' understanding of the rules and to motivate them to carefully read them before class; and with excel files that automatically provide solutions to the homework based on the collected data.

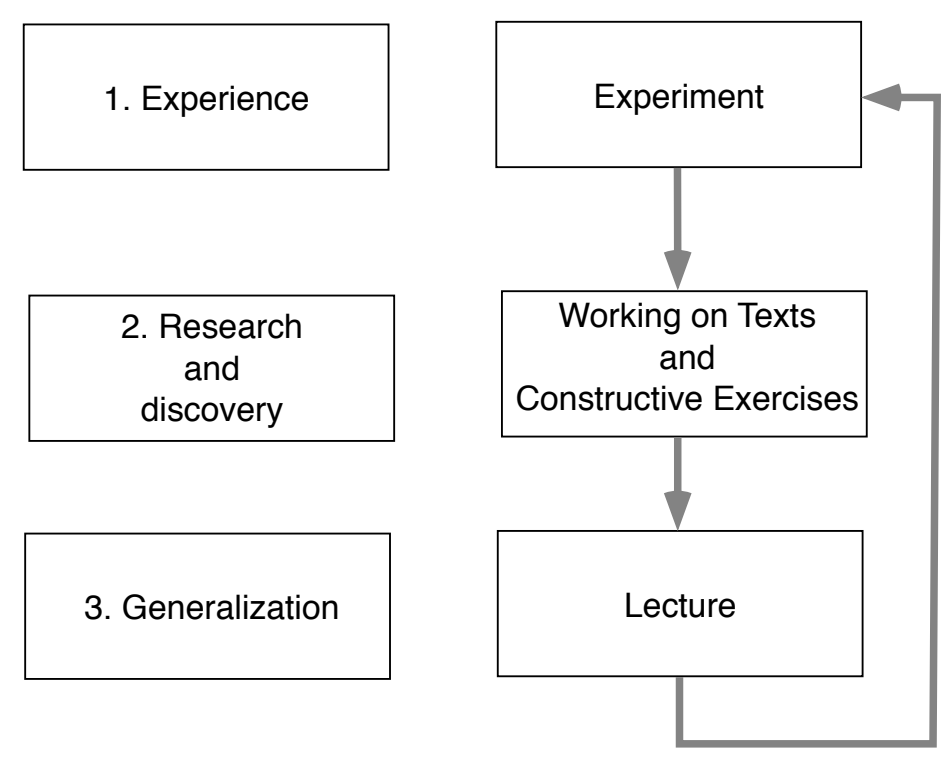

Figure 2. The three stages of our teaching approach. The experiment allows students to experience the economic situation under study and practice their economic intuitions. Working on the constructive exercises and other material, students work on discovering by themselves the main concepts and findings. Finally, the instructor generalizes findings and resolves doubts during the lecture.

For each topic, the material is structured into four groups.

1. An introduction, with detailed instructions and warm-up exercises that prepare students for the experiment. Students must read these instructions and do the warm-up exercises prior to participating in the experiment.

2. A classEx package composed of a four-question quiz based on the warm-up exercises and the actual experiment to be run in the classroom. ${ }^{12}$ The quiz can be used to test students' comprehension just before running the experiment and to motivate them to learn the instructions and work on the warm-up exercises. Students participate with their mobile devices in both the quiz and the experiment. Quizzes are programmed to generate individualized seeds; so different students receive different variations on the same questions. classEx provides instantaneous feedback to students and generates spreadsheets with students' performances in both activities, the quiz and the experiment.

\footnotetext{
12 The Instructor Manual provides the necessary information to run the experiment, as well as tips and comments based on previous experiences.
} 
3. A set of constructive questions based on the data obtained in the experiment. Students ought to work on this homework before the lecture. Problems are designed to guide students step by step in discovering the main concepts by themselves.

4. A discussion section that elucidates the appropriate economic theory and principles. The text connects the experimental results with the theory: It presents the theoretical tools and generalizes the findings to larger markets with a continuum of agents.

\section{IMPLEMENTING THE BERGSTROM-MILLER APPROACH WITH CLASSEX AT UNIVERSITAT POMPEU FABRA: TWO EXAMPLES.}

The Economics Department at Pompeu Fabra University has been using the Bergstrom-Miller approach to teach Principles in Microeconomics since 2008. In the fall of 2016, it adopted classEx to run the experiments paperless. Prior to the adoption of classEx, the experiments were run with pen-and-paper using the forms provided in the Instructor's Manual and data was manually introduced in spreadsheets. The use of classEx substantially reduced the time devoted to running the experiments, allowing for a lengthier discussion of the results. It also eliminated the need of printed-paper and automatized data collection, like participation, benefits, and role allocation. The sessions were run in English, Spanish and Catalan.

Introduction to Microeconomics is offered during the fall quarter to first-year students pursuing a degree in Economics, in Business Administration and in International Business Administration, and also to students following a double degree in economics and law. The course uses eight different experiments covering the following topics: supply and demand, shifts in the supply and demand curves, taxation, labor market and a minimum wage, externalities, monopolies and cartels, short run and long run analysis, and network externalities. Table 1 in the Appendix offers a brief description and some comments for each experiment. As representative examples, we describe in more detail the first two experiments, and report some lessons learned, as well as the descriptive data.

\section{Example I: Supply and Demand Experiment}

The Supply and Demand experiment is well known as it has been widely used in the classroom (Bergstrom and Kwok 2005). Each student is assigned a role either as a seller or as a buyer. classEx automatically assigns roles considering the total number of participating students ${ }^{13}$.

13 classEx implements the matching procedures in Bergstrom and Miller (2000), assuring a well calibrated equilibrium for any class size. 


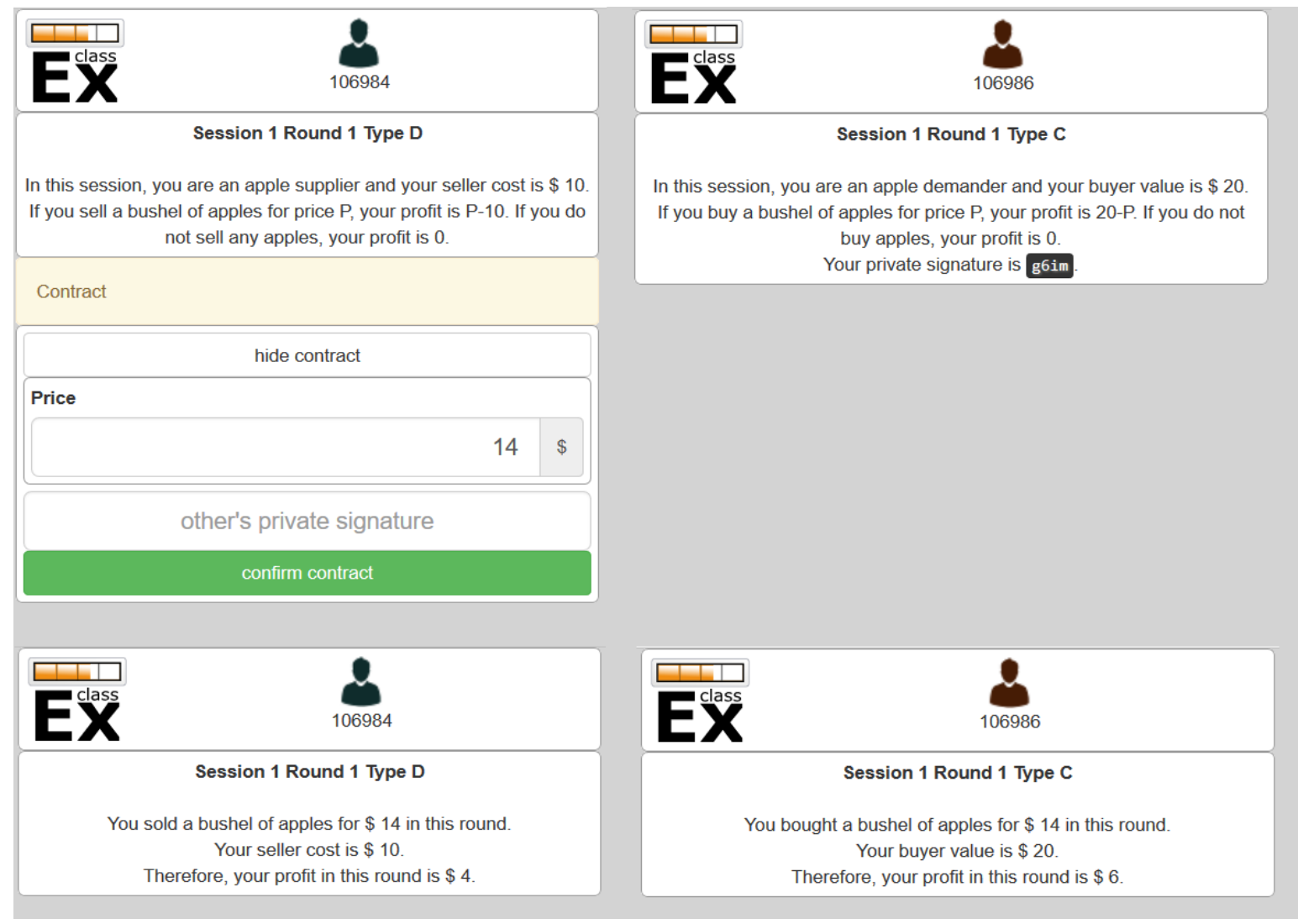

Figure 3. Screenshots for the participant's screens in the apple market. The first row shows the screen during the market phase, the second row the feedback screen. The left column shows a seller with seller cost of $\$ 10$ and the right column a buyer with buyer value of $\$ 20$. The contract is made on the seller side with the signature of the corresponding buyer.

Buyers can buy at most one unit and are informed of their "buyer value" $B V$, that can be either high (\$40) or low (\$20). The top right panel of figure 3 displays the screen of a buyer with a low buyer value. The seller's screen is shown in the top left panel of figure 3 . They can sell at most one unit and are informed of their "seller cost" $S C$, that can be either low $(\$ 10)$ or high (\$30). As can be seen in figure 3, buyer values and seller costs are private information. Students ignore the distribution of buyers and sellers, and do not even know that there are only two types. They should aim at maximizing their profits, which are calculated as the difference between the buyer value and the price $(B V-P)$, or the difference between the price and the seller cost $(P-S C)$, depending on their role. ${ }^{14}$ If a student does not buy or sell, she receives zero profits.

\footnotetext{
14 We let part of the grade depend on profits in order to provide the necessary incentives. In our experience, a small impact of profits in the grade is enough to motivate students, but it is important to design a rule that seems fair while providing the right incentives. For instance, using profits from only some randomly chosen rounds, only revealed a
} 
The experiment has two sessions differing in their distribution of the types. Each session consist of two rounds. It is important to emphasize that classEx allows face to face interaction. Students stand up and move in the classroom, which becomes the trading area, talking to each other and bargaining over the price without sharing their private information. Once they have reached an agreement, the seller enters the price in her mobile device, while the buyer signs the contract providing the private signature shown in his device (see figure 3). Once submitted, the information associated with the contract is made public, projected for the rest of the class (see figure 4). At the end of the round, payoffs are calculated and feedback is privately provided to the students (see bottom row of figure 3). The instructor's screen displays all trades, summary statistics, and the theoretical predictions that can be used in the discussion of the results

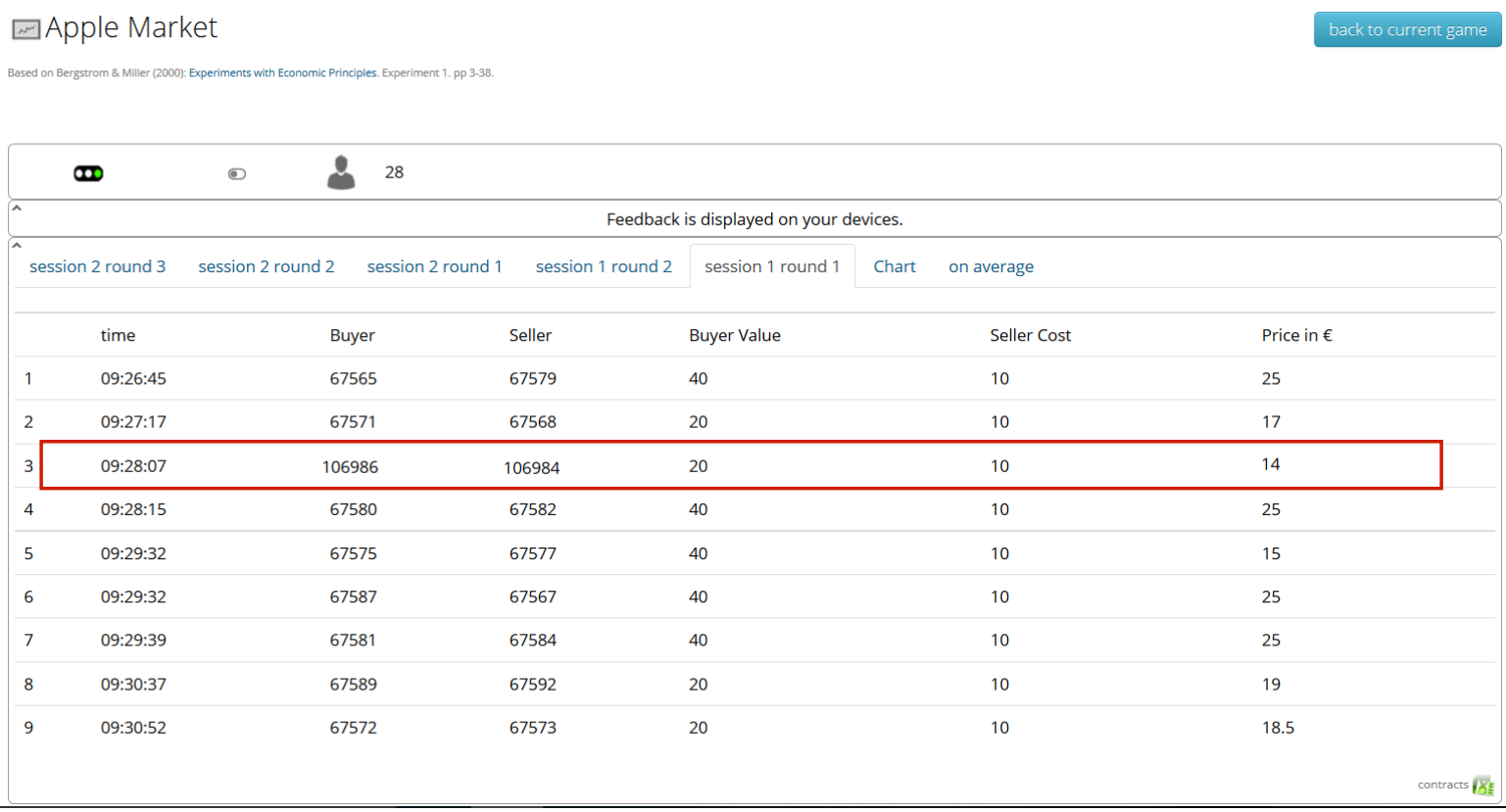

Figure 4. Display of the instructor's screen during the experiment. It shows all contracts and allows participants to check that their contract was correctly registered. It additionally provides the basis for further discussing the results. Other tabs allow the instructor to show descriptive values (average, minimum and maximum prices, and standard deviations) and a chart of the transactions for all rounds. The row in red shows the transaction of the players in figure 3 . Buyer and seller columns show the internal number assigned by the system so other participants cannot identify them. 
The experiment on "supply and demand" was run between the $26^{\text {th }}$ and $29^{\text {th }}$ of September 2016, with a total of 18 sessions and 543 participants. Group size varied from 18 to 37 students. ${ }^{15}$ In the first session, $1 / 3$ of buyers have a high buyer value and $1 / 3$ of sellers have a high seller cost. The equilibrium price is always $\$ 20$, but the equilibrium quantity varies with the size of the group. Figure 5 depicts the demand, supply and equilibrium for a group of 30 students. The figure also shows the resulting averages per group in both rounds of session 1. Overall, average choices are quite close to equilibrium in both rounds.

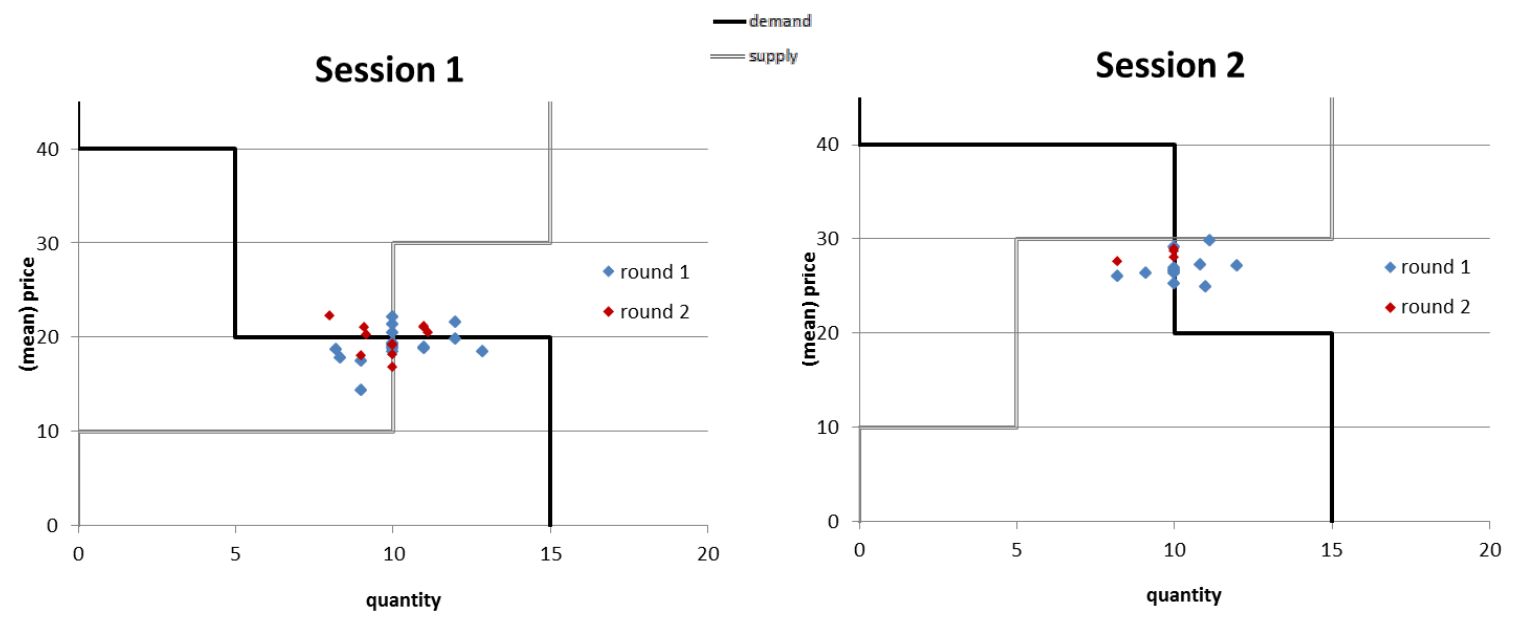

Figure 5. Supply and demand graph for first and second session and average results per group. Quantities were normalized to groups of 30 students and an equilibrium quantity of 10.

Session 1 shows a very fast convergence to the equilibrium price, often happening already in the first round. ${ }^{16}$ In Session 2 the number of sellers and buyers changes. $2 / 3$ of buyers have a high buyer value and $2 / 3$ of sellers have a high seller cost yielding the same equilibrium quantity at a higher equilibrium price of $\$ 30$. Inertia tends to slow down convergence to equilibrium in Session 2, and students tend to repeat prices similar to those observed in Session 1. The slower convergence in Session 2 opens the door to talk about 'sticky prices' and why real world prices do not immediately adjust to equilibrium values. To speed up convergence, we found it helpful to announce that Session 2 represents students moving to a faraway city, emphasizing that the information on prices from Session 1 is of little help. We observe that students quickly

\footnotetext{
${ }^{15}$ In previous years, we ran the experiment with groups of up to 57 students using pen-and- paper, obtaining similar results.

${ }^{16}$ When the equilibrium price is attained in the first round, we skip the second round, gaining extra time for the discussion. This is why the number of observations varies in figure 5 .
} 
understand the dynamics of the experiment and while the first round takes around 4 minutes on average, further rounds require no more than three minutes to be completed (Figure 6). ${ }^{17}$

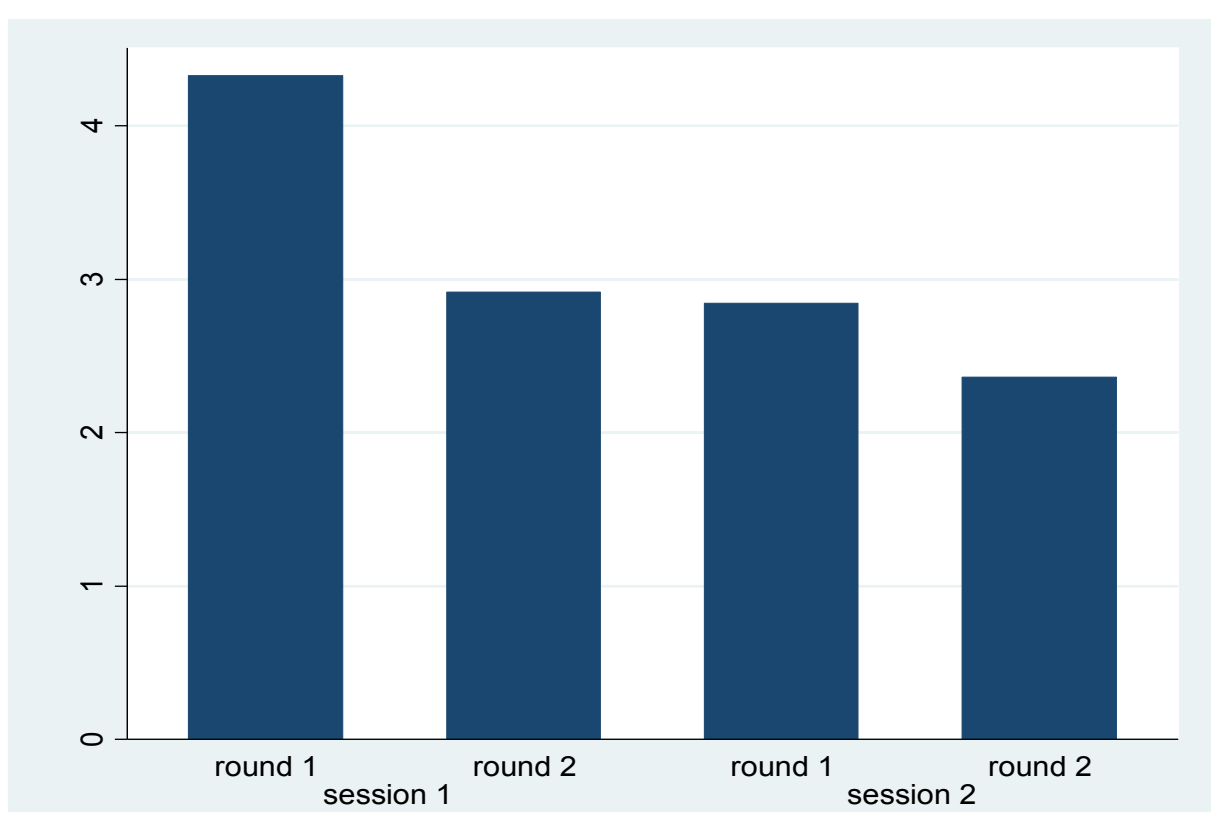

Figure 6. Time (in minutes) for running a round.

The experiment facilitates a deep understanding of the construction of supply and demand, and of the difficult concept of equilibrium. Traditionally, these concepts are learnt in a mechanical manner, often immediately with continuous functions. Markets are presented from a bird-eye's view, overwhelming students with solutions before getting a hand-on intuition Instead, with the help of the experiment, each student "sits" on the demand or the supply function, and the association with reservation prices (that is, the maximum willingness to pay or the minimum willingness to accept) is readily accepted. Similarly, the concept of equilibrium emerges naturally. Students spontaneously return to their seats once they realize they cannot sell or buy. They observe prices converging towards a particular value (the equilibrium price). They learn that not everybody participates in a transaction (at equilibrium) and, more importantly, that the concept of equilibrium price is a construction that approximates (albeit very close) observed market prices. The problem sets and the lectures consolidate these observations and explain the transition from step to smooth

\footnotetext{
17 The total time to run an experiment varies with the number of rounds played. In our experience, thirty minutes are enough for the set up and running two sessions with two rounds each. We often save some time by skipping rounds (see endnote 10).
} 
functions by increasing the number and variety of types. But the deep understanding and the intuition are already there. ${ }^{18}$

The implementation of the apple market with classEx has already been used at nine universities in Spain, Germany and the UK with a total of 59 runs and over 2000 transactions. The average price in session 1 over all those games was $\$ 20.12$, and rose to $\$ 28.57$ in session $2 .{ }^{19}$ This is very close to the equilibrium prices of $\$ 20$ and $\$ 30$ in session 1 and 2 , respectively.

\section{Example II: The Fish Market with Sunk Cost}

This is a variation of the Supply and Demand experiment, with the difference that all sellers have a $\$ 10$ sunk cost and the same marginal cost, equal to zero. The experiment consists of two sessions. A second difference is that sellers have more than one unit to sell in session 2. Each student is assigned a role either as a buyer or as a fisher (seller). Buyers can buy at most one unit and are informed of their "buyer value" $B V$, that can be high (\$25), medium $(\$ 20)$, or low $(\$ 5)$. Sellers' only cost is a $\$ 10$ sunk cost and they are informed of the number of fish they can sell. Buyer values and sellers' catch are private information; while he sunk cost and the zero variable cost are public knowledge. Each student aims at maximizing her profits. For buyers, profits are calculated as the difference between the buyer value and price $(B V-P)$. If a student does not buy, she receives zero profits. Fisher's profits are the sum of all the prices received minus the $\$ 10$ sunk cost. A fisher who does not sell obtains a $\$ 10$ loss. The experiment has two sessions differing in the number of fish caught: one fish per fisher in session 1, and three fish per fisher in session 2.

The experiment on "the fish market" was run between the 26th and 30th of September 2016, with a total of 18 sessions and 538 participants. Group sizes varied from 18 to 36 . In the first session, approximately $1 / 3$ of students are fishers, with the equilibrium quantity being equal to the number of fishers, and the equilibrium price equal to $\$ 20$. Figure 7 (left panel) depicts the demand, supply and equilibrium for a group of 30 students. The figure also shows the resulting averages per group in both rounds of the two sessions. In the first session, traders come close to but slightly below the equilibrium price, as already predicted by Bergstrom and Miller (2000).

${ }^{18}$ We have much anecdotal evidence from the annual student satisfaction surveys conducted by Pompeu Fabra University. Comments of the following type are not uncommon: "The experiments help you interiorize ideas which are later applied to the theory and linked to reality." "The experiments are very helpful for the understating of the concepts, unconsciously bringing our economic vein out." Quotes were translated from Spanish and Catalan by the authors.

${ }^{19}$ Data are taken from the repository in classEx, as of September 20, 2017. 

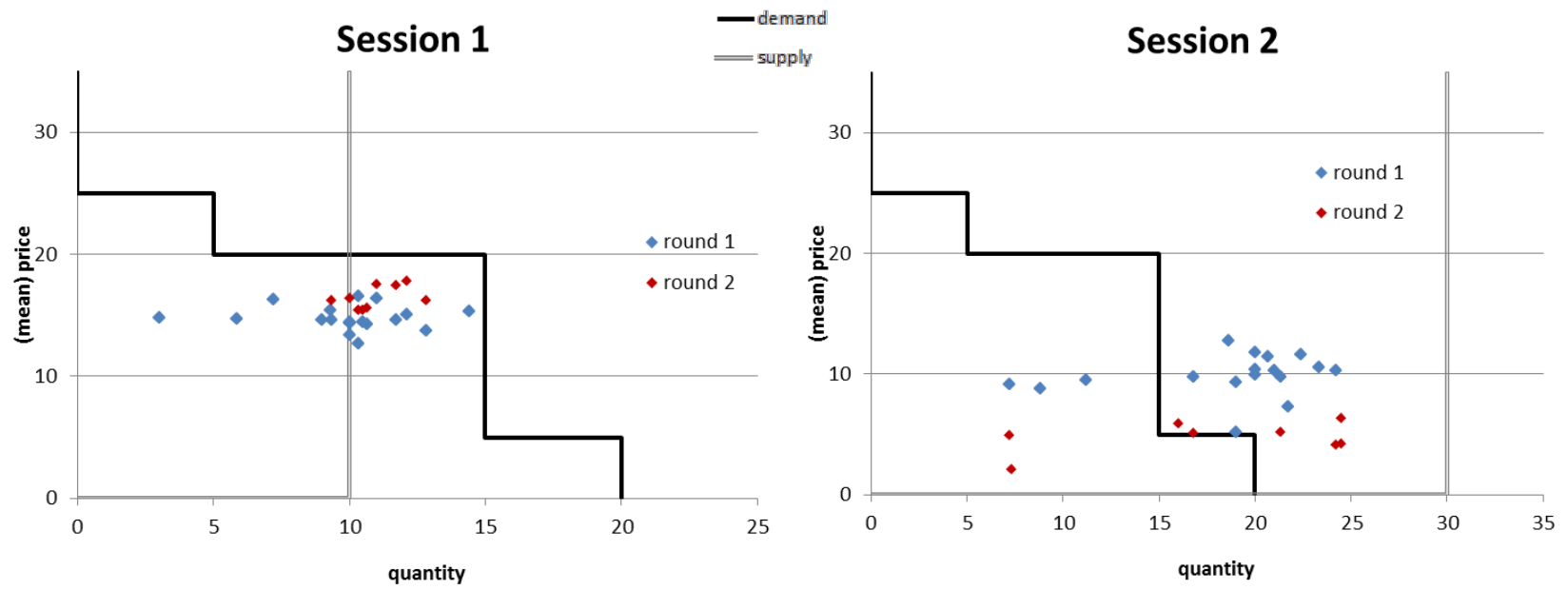

Figure 7. Supply and demand graph for both sessions and average results per group. Quantities were normalized to groups of 30 students and an equilibrium quantity of 10.

In session 2, each fisher catches three fish, flooding the market and dropping the equilibrium price down to $\$ 0$ (Figure 7, right panel). The exact equilibrium price of $\$ 0$ is hard to reach, with average prices remaining strictly positive. However, students' behavior is closer to the equilibrium than the graph may suggest. We observe prices very close to zero in the last transactions of each round, with as many as seven decimal points in some instances. It is a good idea to get students thinking about how fishermen should behave, as many are slow to realize that they are better off selling fish below average cost than not selling them at all. In our courses, we experienced that learning this lesson happens slowly, but pretty surely. And most, if not all, fishers are unable to recover the sunk cost and end up with losses.

These results gave us the opportunity to discuss several topics. First, the concept of sunk cost. Students deduce that a fixed cost higher or a lower than $\$ 10$ would have had no effect on fishers' decisions. Secondly, outcomes show the apparently unintuitive observation that a better catch results in lower profits (even loses) as it shifts the supply upwards. This is a good moment to introduce the distinction between idiosyncratic and generalized shocks. Thirdly, we bring attention to the fact that fishers in Session 2 could have obtained the same profits as in Session 1 if each had destroyed part of their initial catch -two fish- before the market opens. This point is reinforced with newspaper articles on farmers destroying crops and fishers throwing fish back into the sea in order to avoid plumbing prices. Are there alternatives to crop destruction? Would giving the product away or exporting the excessive supply solve the problem? The discussion then extends to common government policies, like the European Common Agricultural Policy (CAP), and its measures to avoid excess supply. Finally, students tend to resist believing in zero prices. We use Phase 1 of the EU Emission Trading System (ETS) as an excellent example, where excess supply of allowances produced a carbon price of zero euro/ton towards the end the period. 


\section{CONCLUSION}

classEx is an online tool that enables experimenters to run lab-in-the-field experiments with mobile devices. It is designed to incorporate experimental needs and practical software implementation issues. Easy access for participants and ready-made games for experimenters facilitate access. Access to games played by others enables researchers to replicate experiments. A flexible architecture guarantees that different forms of games can be implemented in a centralized framework. Standard open formats allow for a broad participation and the standardized layout and caching mechanisms enhance a fast and mobile-adapted communication.

We have been using experiments to teach an Economic Principles course at UPF for the last nine years. In this course, students first experience the situation we want to study, playing a role as an economic agent in an experiment (experiential learning). Economic reasoning and motivation is encouraged by the discussion during and after the experiment, in which students get to exercise their economic intuition. Using the data obtained from the experiments, students are challenged to discover the main concepts by themselves, guided by a constructive set of problems. Working on the constructive problems before the lecture let students identify those issues they do not understand, helping them to focus their attention more efficiently during the lecture. During the lectures, the instructor generalizes the findings and resolves doubts. Finally, we have found that instructors get excited when introduced to the use of experiments in the classroom, resulting in increased motivation and more effective teaching.

\section{REFERENCES}

Ball, S. and C. Eckel. 2004. Using technology to facilitate active learning in economics through experiments. Social Science Computer Review 22(4): 469-78.

Ball, S., C. Eckel, and C. Rojas. 2006. Technology improves learning in large principles of economics classes: Using our WITS. American Economic Review 96(2):442-46.

Bergstrom, T. C., and John H. Miller. 2000. Experiments with economic principles: Microeconomics. Boston, MA: McGraw-Hill Higher Education.

Bergstrom, T. C., and E. Kwok. 2005. Extracting valuable data from classroom trading pits. Journal of Economic Education 36(3): 220-235.

Carter, L. K., and T. L. Emerson. In-class vs. online experiments: Is there a difference? Journal of Economic Education 43(1): 4-18.

Cartwright, E, and A. Stepanova. 2012. What Do Students Learn from a Classroom Experiment: Not Much, Unless They Write a Report on It. Journal of Economic Education 43(1): 48-57.

Dangel H.L. and C.X. Wang. 2008. Student response systems in higher education: Moving beyond linear teaching and surface learning. Journal of Educational Technology Development and Exchange 1(1): 93-104.

Dickie, M. 2006. Experimenting on classroom experiments: Do they increase learning in introductory microeconomics? Journal of Economic Education 37:267-88.

Durham, Y., T. McKinnon, and C. Schulman. 2007. Classroom experiments: Not just fun and games. Economic Inquiry 45(1):162-78.

Emerson, T., and B. Taylor. 2004. Comparing student achievement across experimental and lecture-oriented sections of a principles of microeconomics course. Southern Economic Journal 70(3):672-93. 
Emerson, T., and B. Taylor. 2007. Interactions between personality type and the experimental methods. Journal of Economic Education 38:18-35.

Emerson, T., and B. Taylor. 2010. Do Classroom Experiments Affect the Number of Economics Enrolments and Majors? A Study of Students in the United States. International Review of Economics Education 9(2): 43-58.

Frank, B. 1997. The impact of classroom experiments on the learning of economics: An empirical investigation. Economic Inquiry 35(4):763-69.

Giamattei, M., and J.G. Lambsdorff. 2015. classEx - an online software for classroom experiments. Working Paper. doi: 10.13140/RG.2.1.1007.9848.

Gremmen, H., and J. Potters. 1997. Assessing the Efficacy of Gaming in Economic Education. Journal of Economic Education 28(4): 291-303.

Gremmen, H., and G. van den Brekel. 2013. Do Classroom experiments increase student motivation? A pilot study. European Scientific Journal 2: 346-355. 


\section{APPENDIX}

\section{TABLE 1}

\section{Full List of Experiments Used in the Introduction to Microeconomics Course at Universitat Pompeu Fabra.}

Experiment name

1. Supply and demand

2. Shifting the supply curve and sunk costs

3. A sales tax

4. A minimum wage

5. Entry and exit
Brief description

Buyers and sellers can trade at most one unit of a homogenous good. They privately receive reservation prices. They talk to each searching for the best price. Once they reach an agreement, they submit their transaction that is made public to the rest of the class. Average market prices quickly approach equilibrium prices. There are two sessions varying in the distribution of buyers and sellers. This is the basic experiment and serves as a base model.

Fishers (sellers) have zero marginal cost and a $\$ 10$ non-recoverable fixed cost. There are two sessions differing in the number of fish that each fisher can sell. In session 1, fishers have only one fish to sell and trading is similar to the supply and demand experiment. In session 2, fishers have three fish each. There are more fish than buyers and prices plummet to zero. Students observe that (1) prices do not depend on production cost but on the demand and the supply; (2) sunk costs are irrelevant for fishers' decisions; and (3) a better catch (or yield) may result into a worse situation for fishers.

There are three sessions that share the distribution of buyers and sellers. Session 1 is similar to the supply and demand experiment, albeit with many more different reservation prices. Sessions 2 and 3 introduce a lump-sum sales tax and a lump-sum consumption tax, respectively. Students observe the equivalence of the taxes. Tax revenue is returned in equal shares to all students at the end of the experiment.

Students participate in a labor market. While workers are endowed with a reservation wage, employers receive a discrete production function. In session 1 , employers learn to hire workers only if the wage is below the value of their marginal product. Session 2 imposes a minimum wage (price floor) above the equilibrium wage, generating involuntary unemployment. Session 3 changes production functions to increase labor demand shift the equilibrium wage above the minimum wage.

This experiment has two stages: an "entry" stage and a "market" stage. In the entry stage, students decide in sequential order whether to open or not a restaurant. All restaurants have the same fixed and constant marginal costs and a fixed capacity. Once the number of restaurants is known, the market stage functions as the demand and supply experiment. If there are "too many" restaurants, some will have loses leading to fewer entrants in the next round. If there are "too few" restaurants, there are profits that will attract new entrants in later rounds. Students discuss short vs. long run and their effects on sunk costs and equilibria. 
6. Externalities

8. Network externalities and competing standards
Students "suffer" the cost of pollution as a byproduct of production. Students learn that a Pigouvian tax increases total surplus, complementing the lesson of the excess burden learnt in the a-sales-tax experiment. Asking students to compare the sessions with and without a tax usually results in a unanimous support for a society with a tax and low pollution. However, asking students to vote for the session to be used in computing profits results in several students voting for the no-tax, high-pollution session, as they obtained larger profits. The last session introduces two simultaneous markets: one for the good and one for pollution permits, which are distributed in a fixed amount at the beginning of each round.

Students learn about monopoly pricing, price discrimination and the formation and breakdowns of cartels. Seeing the cartel break apart lead to discussions about collusion, cooperation, and defection. The prisoners' dilemma naturally emerges in the conversation.

Students quickly relate to this experiment, as it is all about E-economics: smartphone apps (like Facebook, WhatsApp, or Pinterest); computer operating systems (Windows, Mac, Linux); or videogame consoles (Nintendo, Xbox, PlayStation). The first session introduces a good that becomes more valuable the more people purchase it. Students observe how a few first hesitant buyers transform into a stream of buyers as the critical mass is attained. The second and third sessions feature fierce competition between competing computer operating systems, in an environment where the strong are likely to devour the weak. 